\title{
Measurements and Simulations of Engineered Ultrasound Loudspeakers
}

\author{
Piotr Bratoszewski, Janusz Cichowski, Andrzej Czyżewski \\ Multimedia Systems Department (MSD), Gdansk University of Technology (GUT) \\ Faculty of Electronics, Telecommunications and Informatics \\ Gdańsk, Poland \\ E-mails: \{bratoszewski, jay, andcz\}@sound.eti.pg.gda.pl
}

Received: 07 December 2014; revised: 26 May 2015; accepted: 07 July 2015; published online: 07 September 2015

\begin{abstract}
Simulation and measurement results of the sound emitted from an ultrasound custom-made system with high spatial directivity are presented. The proposed system is using modulated ultrasound waves which demodulate in nonlinear medium resulting in audible sound. The system is aimed at enhancing the users' personal audio space, therefore the measurements are performed using the Head and Torso Simulator which provides realistic reproduction of the human auditory system. Based on the measurements of the sound emitted from the two parametric arrays of ultrasonic transducers the directivity of the proposed system and the interaural crosstalk characteristics are determined. Application of the system concerns creating a personal audio space for users of mobile platforms, such as notebooks, and applying 3D audio algorithms without the need of using headphones.
\end{abstract}

Key words: sound from ultrasound, parametric array, interaural crosstalk

\section{INTRODUCTION}

High directivity sound is desired in many applications, from the warning auditory sounds in industry, through the advertising spots in the markets, to the gaming industry, mainly in the areas where the sound should be directed only towards one spot without engaging other people than intended listeners of the audio sound. In this paper authors focus on applications related to personal audio space of users who are equipped with a mobile platform such as notebooks or with a personal computer. Achieving a high directivity sound is possible either using large loudspeaker arrays or using arrays of ultrasonic emitters known as parametric acoustic arrays (PAA). In this paper the self-engineered PAA system is used, which is characterized by moderately small aperture and ability to create the long distance spotlight beam of sound. The engineered PAA was modeled and simulated firstly using a specialized ultrasound simulation tool FOCUS, therefore various modeling results of the array are presented herein. Furthermore, using a highly specialized Head and Torso Simulator (HATS) produced by Brüel\&Kjær, human perception of the sound emitted by the proposed PAA system can be examined. Having adequate measurement results, the authors propose a number of possible applications of such a system.

The paper is organized as follows. In the next section the theory behind the parametric acoustic arrays is presented. Section 3 describes the engineered hardware used for the research. Sections 4, 5 and 6 outline the simulations of the proposed array, measurement methodology and the results, respectively. Finally, Section 7 concludes the paper.

\section{THEORETICAL ASPECTS OF THE PARAMETRIC ACCOUSTIC ARRAYS}

The parametric acoustic array was first introduced theoretically by Westervelt in 1963 [1]. The first application of PAA in the air by Yoneyama et al. is dated to 1983 [2]. PAA utilizes nonlinear acoustic phenomena to make the sound audible. Acoustic nonlinearities occur due to the fact that 

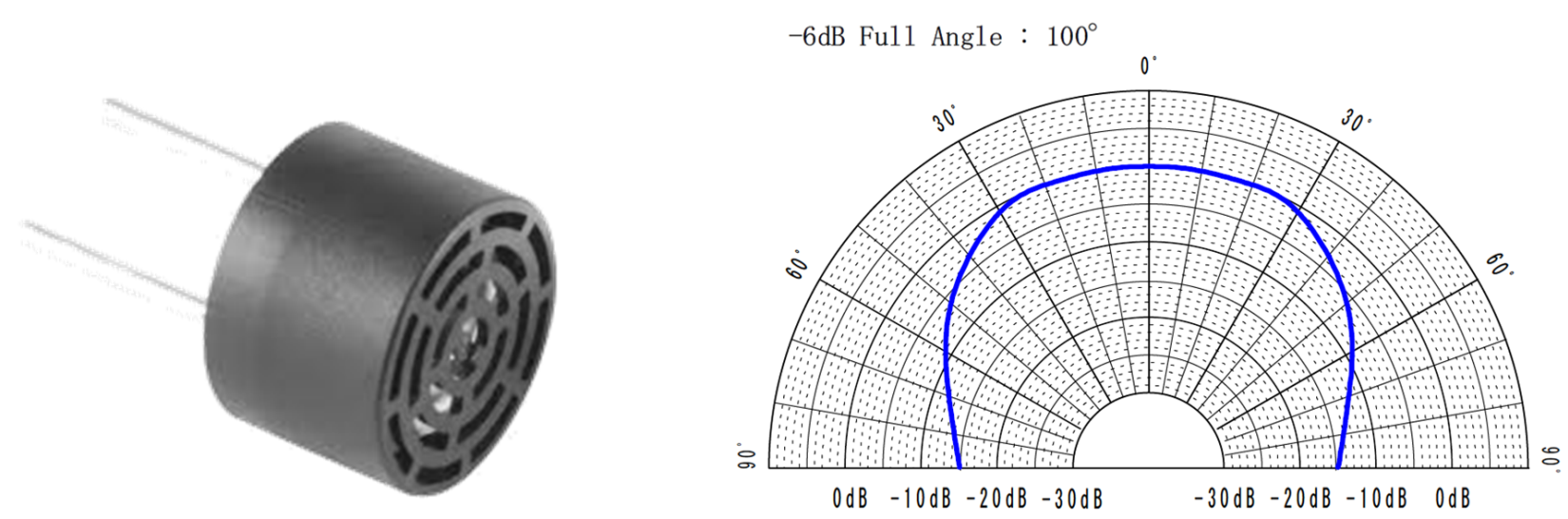

Fig. 1. T4010A ultrasound transmitter enclosure and directional response [9]

it takes less time for the air particles to compress than to restore to their starting points, and that waves of different frequencies interact with each other. Parametrically generated sound fields can be theoretically modeled by the KhokhlovZabolotskaya-Kuznetsov equation [3], which combines nonlinearity, dissipation and diffraction of a directive sound beam. The equation is modeled as follows:

$$
\frac{\partial^{2} p}{\partial z \partial t^{\prime}}=\frac{c_{0}}{2} \nabla_{\perp}^{2} p+\frac{\delta}{2 c_{0}^{3}} \frac{\partial^{3} p}{\partial t^{\prime 3}}+\frac{\beta}{2 \rho_{0} c_{0}^{3}} \frac{\partial^{2} p^{2}}{\partial t^{\prime 2}}
$$

where $p$ is the sound pressure, $c_{0}$ is the sound speed, $\rho_{0}$ is the medium density, $\delta$ is the sound diffusivity related to sound absorption, $\beta$ is the nonlinearity coefficient. Moreover, $\nabla_{\perp}^{2}=\partial^{2} / \partial x^{2}+\partial^{2} / \partial y^{2}$ is the Laplacian that operates in the $x-y$ plane, perpendicular to the axis of the beam, $t^{\prime}=t-z / c_{0}$ is the retarded time.

Gan et al. [4] reviewed several techniques that have been introduced to date in the field of parametric acoustic arrays for achieving audible sound. Kamakura and Aoki [5] presented profound considerations on both modulation and hardware aspects of using the parametric array in the air.

Westervelt suggested using two waves with primary frequencies of $f 1$ and $f 2$, where $f 2$ is larger than $f 1$. Those waves combine in the air and result in sound of frequency $f=f 2$ and $f=f 2-f 1, f 2+f 1$ with halved intensity. Only the differential wave can be heard by the human. The sum wave and the carrier wave remain inaudible due to their high frequency and fast attenuation in the air. Worth mentioning are the practical experiments performed by Gudra and Opielinski. Authors present the results of measurements of the differential wave obtained by using two types of transducer matrices in an anechoic chamber [6].

Yoneyama presented the implementation of PAA which used the amplitude modulation (also known as double sideband amplitude modulation) technique where the $40 \mathrm{kHz}$ sinusoidal carrier was used, which lies far beyond the human hearing range but introduces a high level of total harmonic distortion (THD) [2]. In DSBAM the high frequency carrier interacts with upper and lower side-band components in a nonlinear manner. In order to reduce the level of THD Kamakura et al. presented the square-root AM (SRAM) method [7].

Hitherto, from all known modulations, the single sideband amplitude modulation (SSB-AM is characterized by the lowest level of THD and the highest power efficiency). However, the main drawback of SSB-AM is related to the envelope errors in case where the emitted signal consists of a broadband signal (such as speech or music) which modulates the carrier. To overcome this issue, Croft et al. [8] proposed application of the recursive SSB-AM (RSSB-AM).

The present authors are using the PWM type of modulation where the carrier signal is a rectangular pulse wave whose pulse width is modulated by the amplitude of the desired input signal. Further information concerning authors' solution will be presented in the next section.

\section{DEVELOPMENT OF THE ULTRASOUND LOUDSPEAKER}

The scientific and technological reports corresponding to the sound generated using the ultrasonic transducers configured in the form of PAA motivated authors to develop a test setup that enables examining the aspects of creating personalized sound space for mobile and multimedia applications. According to the theoretical basis mentioned in the previous section, the stereo system equipped with the pair of the ultrasound loudspeakers mounted on the servomechanisms were developed.

A single ultrasound loudspeaker contains an array of ultrasound transducers and the array's driver. The essential elements of the device are the piezoelectric transducers (PZT) type T4010A [9] manufactured by Nippon Ceramic Co. of Japan. The diameter of a single transducer is equal to 10 $\mathrm{mm}$, and its height equals $7 \mathrm{~mm}$. This type of a transducer was selected due to the satisfactory balance between its price (less than 1 EUR) and stability of the parameters. A single 
transducer generates a sound pressure level equal to $120 \mathrm{~dB}$, measured at the distance of $30 \mathrm{~cm}$ in front of the transducer. The transducer enclosure and the directional response characteristics provided by manufacturer are shown in Fig. 1.

In the authors' solution, 18 ultrasound transducers are mounted on the FR4 laminate in the form of a honeycomb, which enables reduction of the spacing between the transducers. The physical aperture of the constructed PAA is presented in Fig. 2.

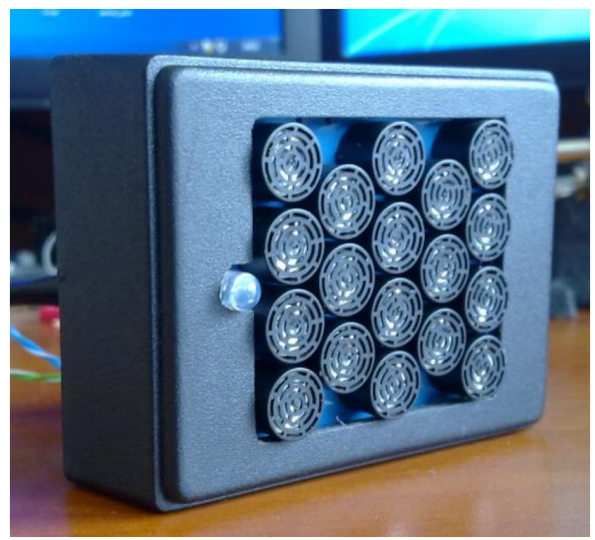

Fig. 2. Physical aperture of the designed parametric array

The parametric array is an electro-acoustic part of the developed ultrasound loudspeaker that transduces a signal from the electronic circuits to the acoustic domain. The transducers resonant frequency oscillates around $40 \mathrm{kHz}$. The audio signal may be audible from the ultrasound array only if the specific type of signal processing is applied. There are several different types of signal modulation which were shortly described in the introductory part of this paper. The authors are mainly focused on the possible PAA applications and technology functionalities, not particularly on the development of a novel type of a parametric loudspeaker. Hence, the electronic part of the designed loudspeaker is based on the available driver F100B [10] manufactured by Nippon Ceramic. The electronic circuit of the F100B driver was analyzed, whereas the analysis enabled defining the schematic diagram presented in Fig. 3.

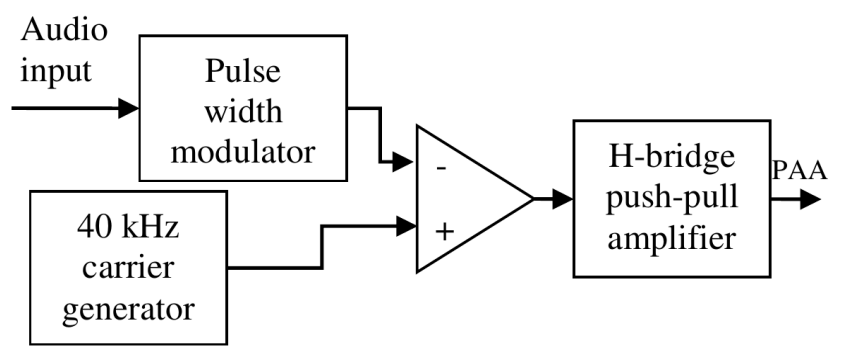

Fig. 3. Block diagram of the parametric array driver
The utilized parametric array driver is not oriented on the quality of the audio signal reproduction, nevertheless it enables the authors to evaluate the possible applications of the system based on the parametric acoustic array. The pulse width modulation technique combined with the PAA was reported by Miura [11]. The significant disadvantage of the PWM modulation is the introduction of the harmonic distortion; on the other hand, it is easy to make a prototype, because there are numerous integrated circuits ICs available on the electronic market.

The last element of the developed loudspeaker is the rotation mechanism which contains the servomechanism and the microcontroller unit that is responsible for the communication with the computer using the USB protocol. The controller is able to handle up to 10 servomechanisms, whereas the angular range of rotation is equal to $180^{\circ}$.

\section{MODELING AND MEASUREMENTS OF THE ULTRASOUND LOUDSPEAKER}

The electro-acoustic elements of the system (transducer and array) were modeled and simulated. For that purpose, authors used the MATLAB framework called Fast ObjectOriented C++ Ultrasound Simulator FOCUS [12] developed by the Michigan State University. The software enables creating models of the specific acoustic apertures and simulating the near-field as well as far-field propagation. The simulated radiation pressure of the single T4010A is presented in Fig. 4.

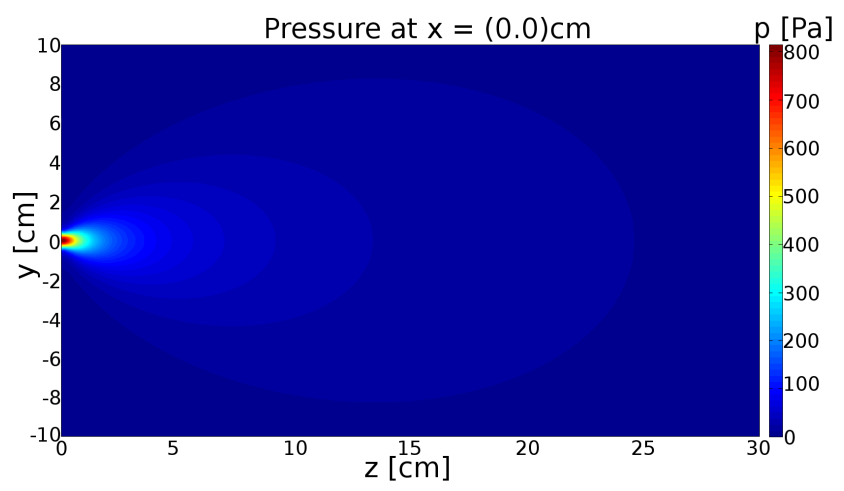

Fig. 4. Radiation pressure of a single transducer at $X=0 \mathrm{~cm}$

The mathematical model of a single transducer is consistent with the parameters reported in the datasheet [9]. The distribution pressure emitted from the PZT was analyzed in the geometric center of the transducer in the range of 0 to $30 \mathrm{~cm}$.

The model of the designed parametric array was also created, basing on the modeled aperture various simulations were performed and described in this Section. The model depicted in Fig. 5 consists of the ultrasonic transducers analyzed as the circular pistons. 


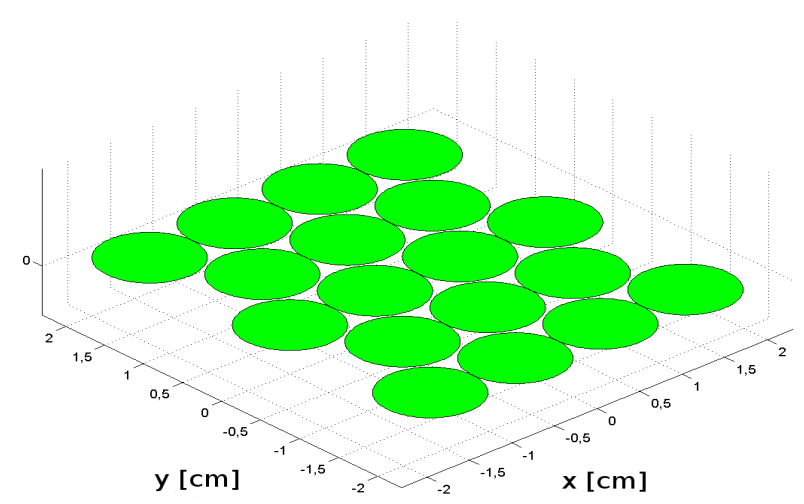

Fig. 5. Mathematical model of the developed parametrc acoustic array

The simulations were applied according to the ultrasound propagation in the air. The result of the simulation in the $X Z$ plane in the geometric center of aperture along $Y$ axis in the range of 0 to $60 \mathrm{~cm}$ is presented in Fig. 6 .

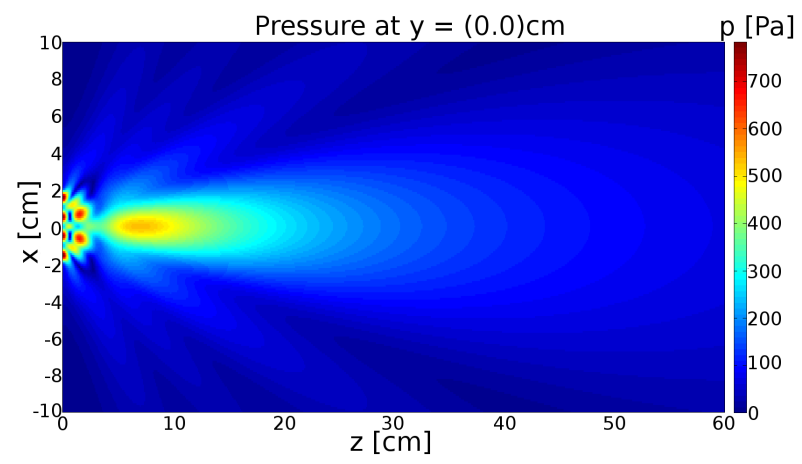

Fig. 6. Radiation pressure of a parametric array $Y=0 \mathrm{~cm}$ (geometric center of array)

The distribution of sound pressure emitted from the parametric array is significantly more directive and presents higher efficiency on the propagation axis in comparison with a single ultrasound transducer. Because of unequal vertical and horizontal dimensions occurrence of the developed and modeled aperture the radiation pressure was analyzed in the $\mathrm{YZ}$ plane in the geometric center of aperture along $X$ axis. The result of the simulation is presented in Fig. 7.

There are expected differences in the radiation patterns for different aperture orientations. However, for both cases the main radiation lobe represents strong directivity along $Z$ axis.

Furthermore, authors took into account the problem of the ultrasound propagation in the near and far fields. The sound pressure fields for both near and far variants were computed using an appropriate mathematical apparatus offered by FOCUS software. The results of the simulation in the $X Y$ plane at the distance of $1 \mathrm{~cm}$ (near field) for the modeled parametric array is presented in Fig. 8.

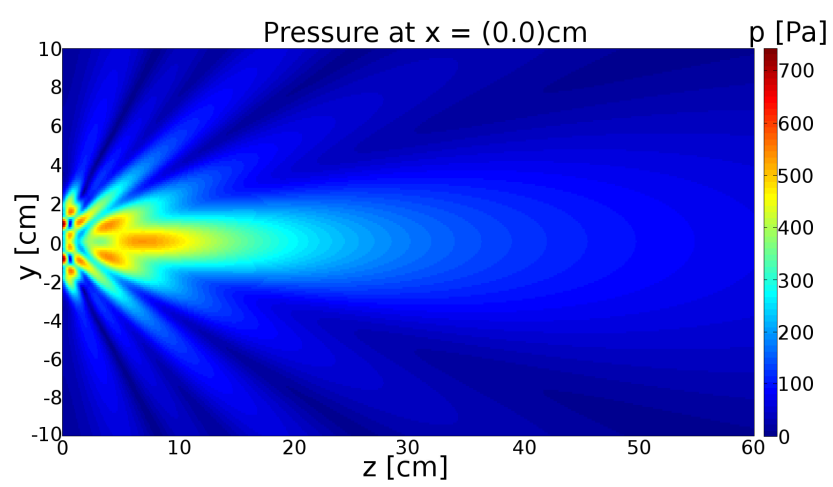

Fig. 7. Radiation pressure of a parametric array $X=0 \mathrm{~cm}$ (geometric center of array)

The results of the simulation in the $X Y$ plane at the distance of $60 \mathrm{~cm}$ (far field) for the modeled parametric array is presented in Fig. 9.

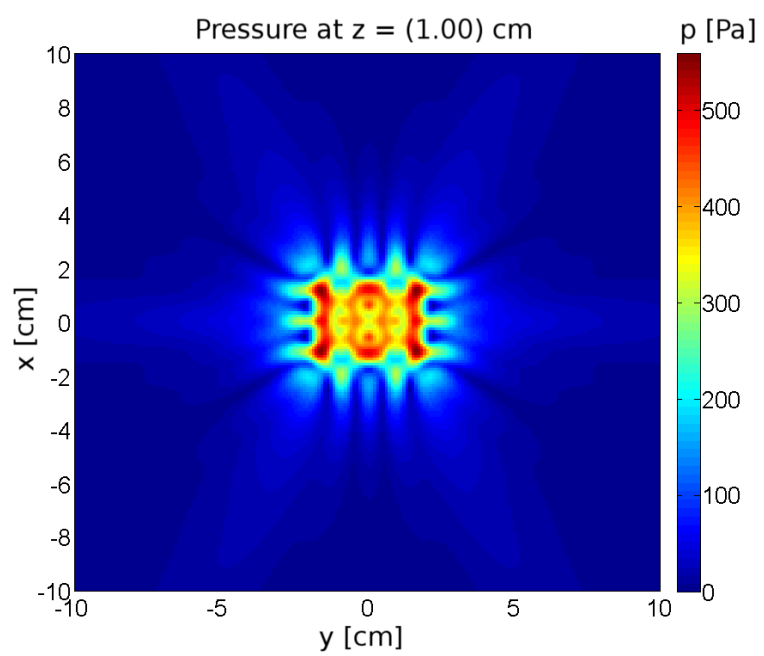

Fig. 8. Near field radiation pressure of a parametric array $Z=1 \mathrm{~cm}$

The results of the simulations for both near and far fields are consistent with the theoretical aspect of the propagation medium (air) nonlinearity. The pressure radiated from the transducers in the near field is significantly greater than in the far field and the radiation pattern corresponds to the aperture arrangement. Due to the nonlinear acoustic phenomena the pressure in the far field significantly decreases; however, at the distance of $60 \mathrm{~cm}$ the maximum radiated pressure is still considerably high and equal to ca. $70 \mathrm{~Pa}$. That may be translated using mathematical formula given by Eq. 2 :

$$
L_{\mathrm{SPL}}=20 \log _{10}\left(\frac{p}{p_{0}}\right)[\mathrm{dB}]
$$

where $p_{0}$ is the reference sound pressure equal to $20 \mu \mathrm{Pa}$. For $70 \mathrm{~Pa}$ the sound pressure level is equal to ca. $130 \mathrm{~dB}$ SPL. 


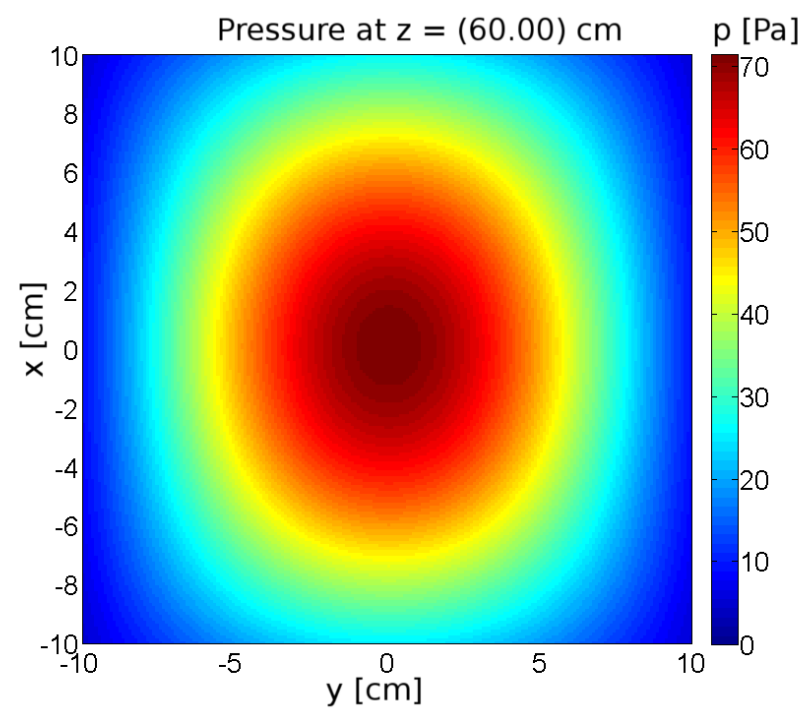

Fig. 9. Far field radiation pressure of a parametric array $Z=60 \mathrm{~cm}$

The far-field pressure radiated by the PAA is distributed in the area within the size of $10 \mathrm{~cm} \times 10 \mathrm{~cm}$ as shown in Fig. 9 .

The results of PAA simulation computed in the MATLAB employing the FOCUS framework were verified in an anechoic chamber. Frequency characteristics and directivity of the designed loudspeaker were measured using the PULSE measurements system produced by Brüel\&Kjær. The performed measurements considered spectral and directional responses of the PAA. Moreover, the different input signal amplitudes weighed as RMS were considered $(50 \mathrm{mV}, 100 \mathrm{mV}$, $500 \mathrm{mV}$ and $1 \mathrm{~V}$ ). The frequency response was measured with the microphones placed at the distances of: $0.5 \mathrm{~m}, 1 \mathrm{~m}$ and 2 $\mathrm{m}$ in front of the loudspeaker. The stepped sine was used as a testing signal for each measurement, whereas duration of the test signal was fixed as equal to $1.6 \mathrm{~s}$. The analysis of the frequency response was restricted to the $100 \mathrm{~Hz}$ to $20 \mathrm{kHz}$ bandwidth. The results are presented using the logarithmic frequency scale on the $X$ axis and dB SPL on the $Y$ axis as in Fig. 10-12.

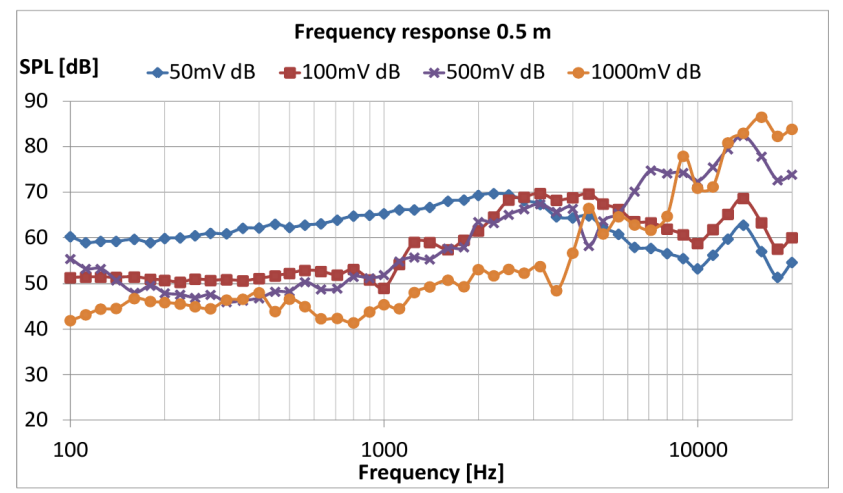

Fig. 10. Frequency response measured at $0.5 \mathrm{~m}$

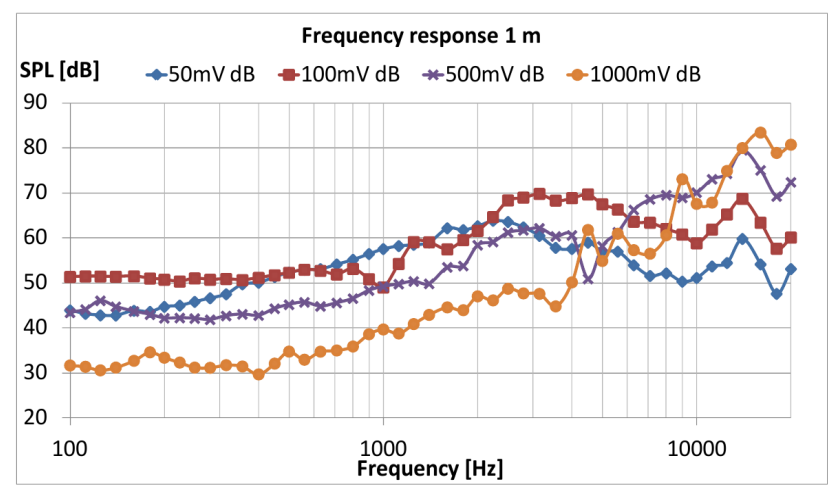

Fig. 11. Frequency response measured at $1 \mathrm{~m}$

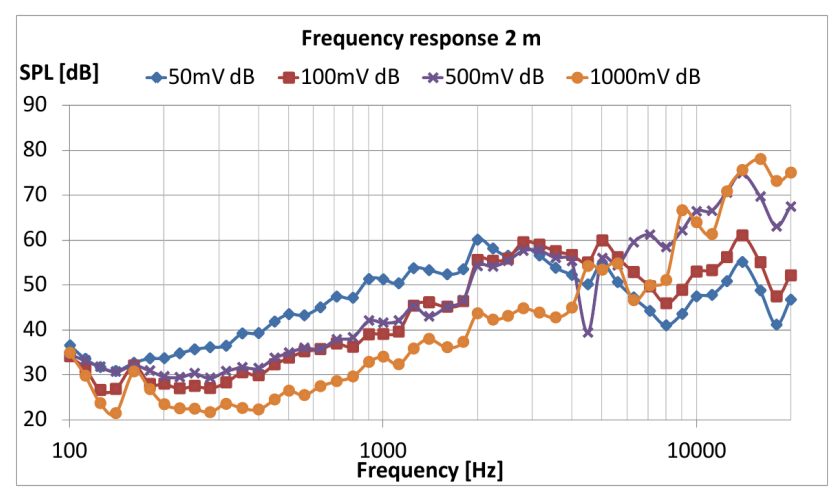

Fig. 12. Frequency response measured at $2 \mathrm{~m}$

The obtained results prove that it is very easy to overdrive the constructed ultrasound loudspeaker. The most linear frequency response was obtained at distance of $0.5 \mathrm{~m}$ from the PAA using input signal with RMS level equal to $50 \mathrm{mV}$. PAA 
presents higher efficiency on higher frequencies; on lower frequencies the loudspeaker is practically linear, but less efficient. It is worth noting that the higher the input level is set, the smaller the efficiency of the loudspeaker after overdriving is seen. The $\pm 6 \mathrm{~dB}$ bandwidth is observed in the frequency response obtained at $1 \mathrm{~m}$ in front of the loudspeaker using $100 \mathrm{mV}$ excitation. The $\pm 6 \mathrm{~dB}$ bandwidth falls within the range of $1250 \mathrm{~Hz}$ to $10 \mathrm{kHz}$, the $\pm 3 \mathrm{~dB}$ bandwidth is in the band ranging from $2 \mathrm{kHz}$ up to $8 \mathrm{kHz}$. Since RMS of the signal is equal to $100 \mathrm{mV}$ or alternatively to $200 \mathrm{mV}$, it is possible to achieve the compromise between efficiency and linearity in the narrow bandwidth. The analysis of the total harmonic distortions in frequency range from $100 \mathrm{~Hz}$ to $10 \mathrm{kHz}$ is presented in Fig. 13.

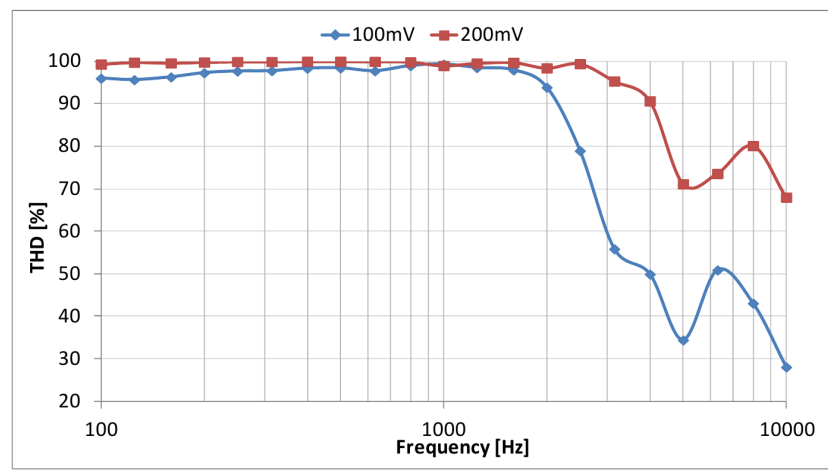

Fig. 13. Total harmonic distortion in respect of signal's RMS

The THD plot presents a very high distortion level in the frequency band from $100 \mathrm{~Hz}$ to $2 \mathrm{kHz}$. The smallest THD rate is observed in the range from $5 \mathrm{kHz}$ to $10 \mathrm{kHz}$ and it is equal to $34 \%$ for the signal with RMS of $100 \mathrm{mV}$ and $70 \%$ for the signal with RMS of $200 \mathrm{mV}$. In the future applications it is possible to reduce THD using another type of modulation, as well as introduce pre-processing of the desired signal to maintain it in the range of the low THD.

The directional response was measured for both orientations of the loudspeaker, because of its rectangularity (horizontal and vertical). The directional responses were analyzed using the sine signal with the RMS level equal to $100 \mathrm{mV}$ or alternatively to $200 \mathrm{mV}$ and frequencies: $1 \mathrm{kHz}, 2 \mathrm{kHz}$, $4 \mathrm{kHz}$ and $8 \mathrm{kHz}$. The directional responses are shown for the loudspeaker placed horizontally (Fig. 14) and vertically (Fig. 15).

The obtained measurement results match the mathematical simulations. The main lobe width of the designed ultrasound array is ca. $20^{\circ}$. Specific frequencies propagate in a different way, the frequency of the signal influencing the signal directivity, similarly as in case of electromagnetic loudspeakers. The designed loudspeaker allows emitting the audio signal with the increased directivity; on the other hand, the useful bandwidth is limited to the range from $2 \mathrm{kHz}$ to $8 \mathrm{kHz}$. The proposed use cases are presented and analyzed in subsequent sections.

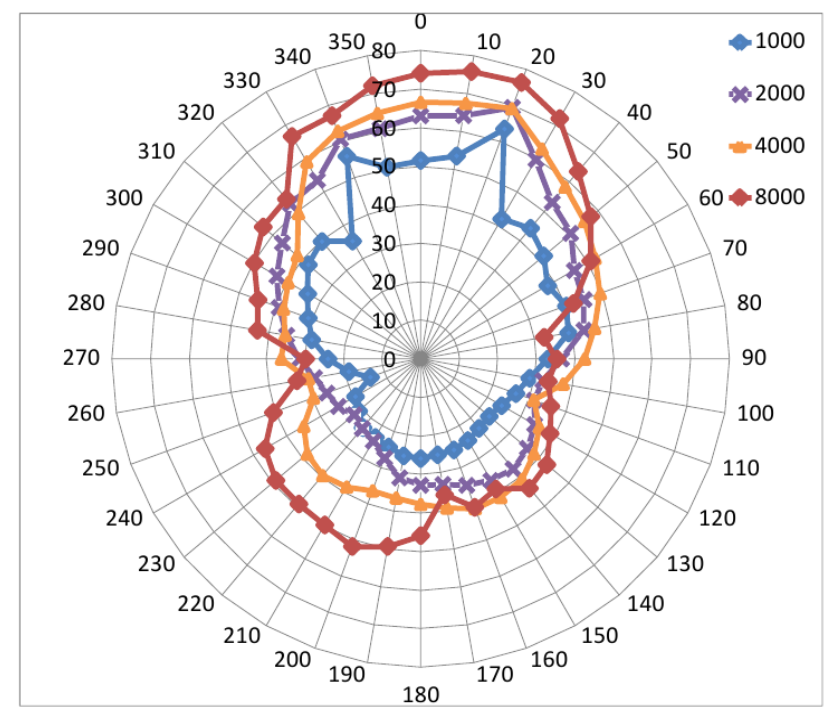

Fig. 14. Directional response in horizontal loudspeaker orientation

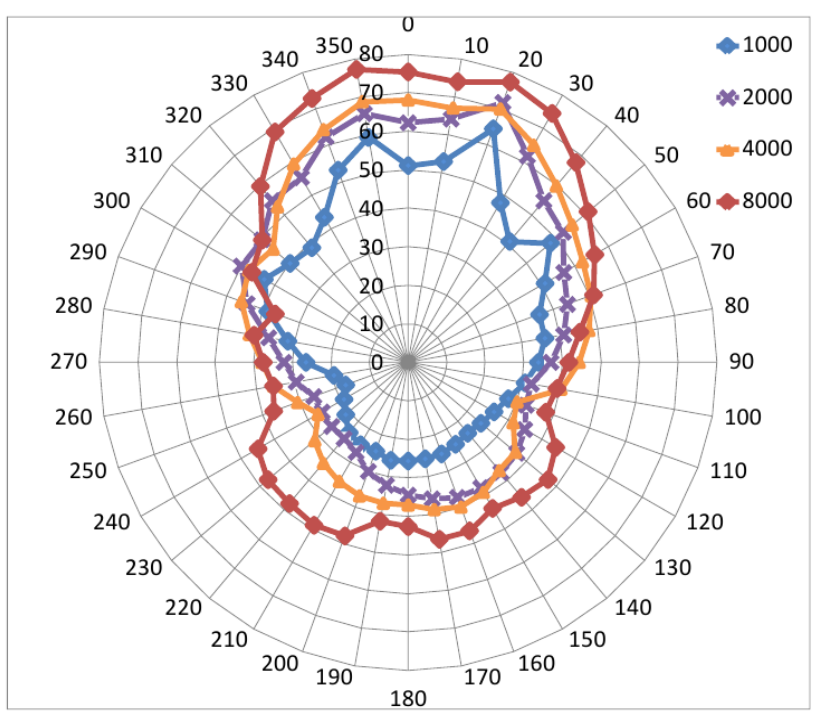

Fig. 15. Directional response in vertical loudspeaker orientation

\section{INTERAURAL CROSSTALK MEASUREMENTS}

Due to the high directivity of engineered ultrasonic loudspeakers and with regard to the idea of creating a personal audio space, the measurements of interaural crosstalk and crosstalk between two listeners were conceived. Fig. 16 presents the measurement setup for interaural crosstalk, where the testing signal is generated by a single speaker, whereas two microphones placed in the ears of the HATS phantom head are the receivers. 


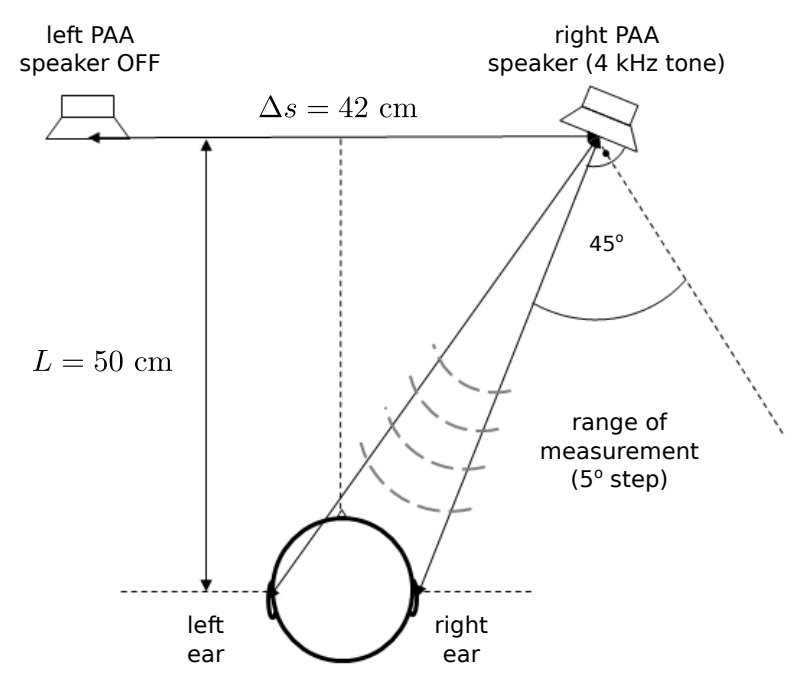

Fig. 16. Interaural crosstalk measurement configuration

The single loudspeaker was employed with the assumption that the presented configuration is symmetrical. A $4 \mathrm{kHz}$ tone was used as a testing signal in measurements, with the distance between the speaker and the listener equal to $55 \mathrm{~cm}$. The interaural crosstalk for a single tone was measured with 5 degree step in the range from 0 to 45 degrees, where zero degrees means that the speaker is aiming at the nose of the listener. The results are presented in Fig. 17. The line with triangle marks represents the signal attenuation registered in the ear that is more distant from the loudspeaker. Frequency axes in the plots presented below are scaled from $1 \mathrm{kHz}$ to
$20 \mathrm{kHz}$ in order to enhance the readability and due to the fact that the test signals fall within this range.

As noted on the graphs, the highest value of the interaural amplitude difference equals $16.20 \mathrm{~dB}$ for the loudspeaker rotation angle equal to $35^{\circ}$. For this rotation angle the signal level in the closer ear (right) equals $72.68 \mathrm{~dB}$. The best balance between the received signal level and the interaural attenuation was achieved for the rotation angle equal to $20^{\circ}$; for this setup signal separation between the ears equals 15.28 $\mathrm{dB}$ and the host signal level in the closer (right) ear equals $79.83 \mathrm{~dB}$.

According to these measurements, the spatial filtration using the ultrasonic loudspeaker allows achieving the signal attenuation level of ca. $15 \mathrm{~dB}$. An additional signal processing (e.g., crosstalk cancellation algorithms [13]) might be used for enhancing the separation between the ears. The measurements were carried out in an anechoic chamber; however, in practical applications some problems with signal reflection may occur. In Fig. 18 the interaural attenuation level with respect to the loudspeaker angle is presented.

\section{MEASUREMENTS OF CROSSTALK BETWEEN LISTENERS}

Two approaches to measurement of the crosstalk between two listeners localized side-by-side are proposed: first, with loudspeakers targeted directly on listeners' heads (en face) with the shortest wave paths presented in Fig. 19 a), and second scheme when the loudspeakers wave paths are travelling crosswise (cross), as shown in Fig. 19 b). Presented measurement results are related to the use case of different audio signals being transmitted to different listeners, e.g. watching a movie simultaneously in two different languages.
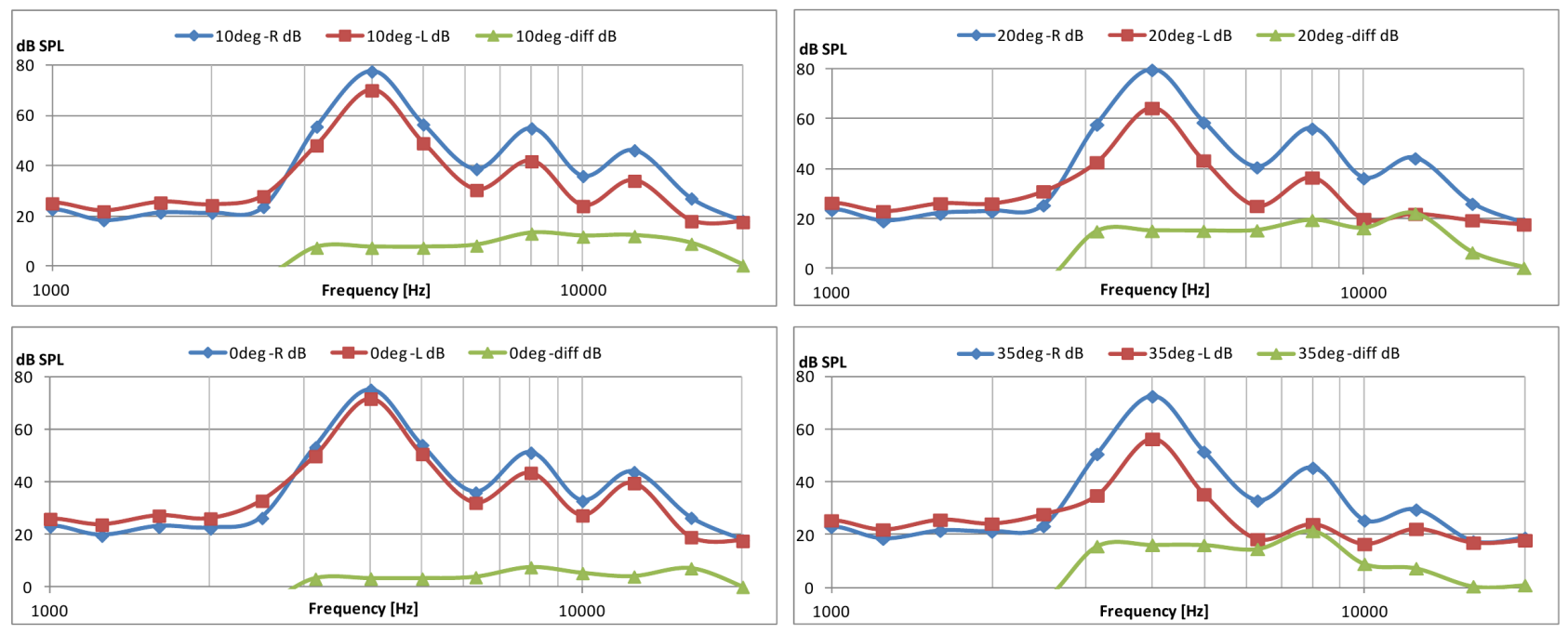

Fig. 17. Graphs representing signal levels in both ears and their difference based on the loudspeaker angle relative to users head 


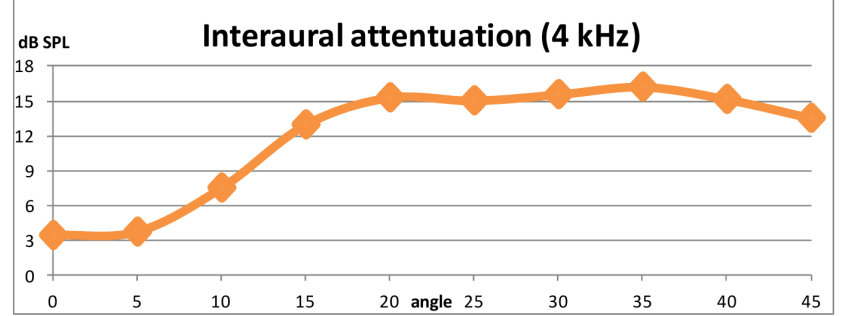

Fig. 18. Interaural attenuation level with respect to the loudspeaker angle
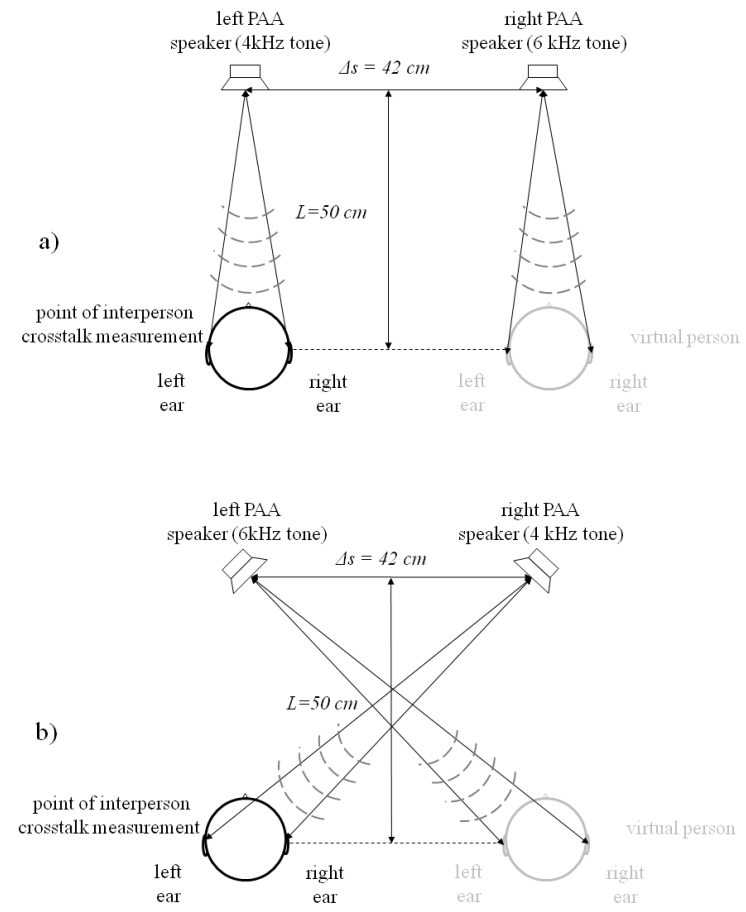

Fig. 19. Two approaches for measuring crosstalk between two persons: a) speakers en face, b) speakers crosswise

In those measurements two signals were used, for the left listener the $4 \mathrm{kHz}$ tone was transmitted and simultaneously for the right virtual listener $6 \mathrm{kHz}$ tone was emitted. For these measurements a lack of reflections from listeners was assumed. Results of measurements for both configurations are presented in Fig. 20 and Fig. 21.

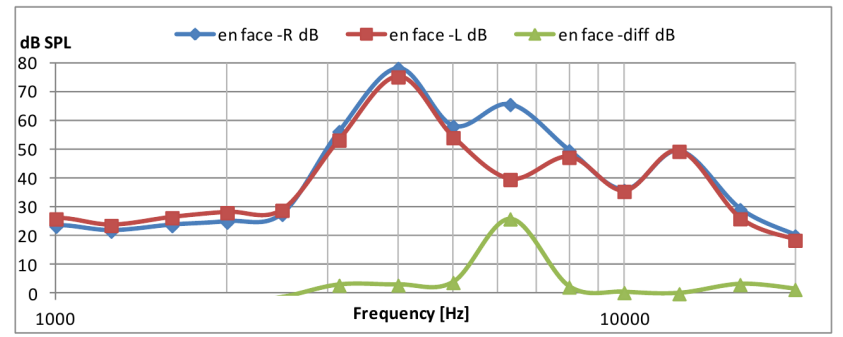

Fig. 20. Crosstalk between two listeners in en face setup

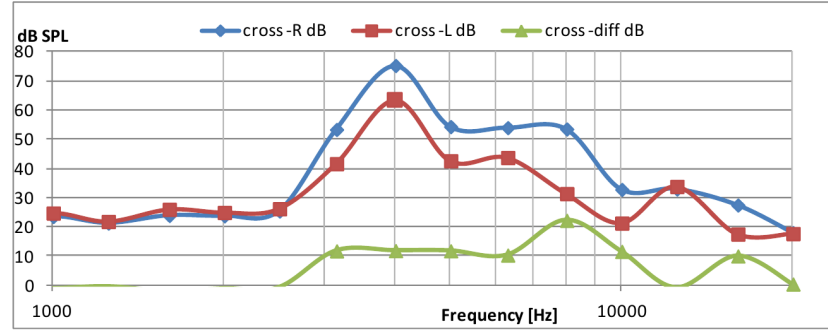

Fig. 21. Crosstalk between two listeners in crosswise setup

Results of an en face measurements present significant signal leakage into the right listener's ear, because of the beam width. In the crosswise configuration the leakage was reduced, but there were observable differences between the listener's ears caused by the location of the listener in parallel to a PC monitor, and not to the loudspeaker surface.

\section{DISCUSSION AND CONCLUSIONS}

Extensive simulations and measurements of the engineered ultrasonic loudspeakers were presented and analyzed. The simulations were performed using the specialized software library Focus, whereas the measurements were carried out in the anechoic chamber. The developed experimental setup introduces large audio signal degradation due to the simplistic approach for the ultrasound signal modulation. The future work in this area is planned, since the utilization of a more sophisticated modulation technique is needed in order to bring better sound quality. The directional response of the engineered PAA is satisfactory, nevertheless the improvements are possible by the use of the beamforming techniques. Measurements proved that the sound from ultrasound speakers can be used for enhancing the personal audio space. Regarding the tolerable level of interaural crosstalk this method of sound reproduction might be used to send the spatial cues to the listener's ears for enhancing the feeling of immersion into the 3D sound space. The level of crosstalk between two listeners in the crosswise approach enables users to listen to different audio contents at the same time. Authors might see the application of the presented system in smart television systems, where users could simultaneously watch two videos and accompanying audio contents on a single TV set. The TV set shall use the 3D techniques to provide each user with his or her own visual content and the PAA using its high directivity properties would provide the individual audio content. According to a relatively narrow frequency band of PAA, the possible application of such a system is limited mostly to the content occupying the bandwidth of a typical human voice (eg., movie dialogues, VoIP systems) and to spatial cues of sound effects in video games or movies. To meet the users' requirements for the future application of such a system a deep analysis of potential use cases is needed. 
The subjective tests oriented on the analysis of interaural crosstalk and crosstalk between the two listeners using real signals such as movie dialogues, movie effects, music, etc. is to be carried out.

\section{Acknowledgment}

This research was partially funded by grant No. PBS1/B3/16/2012 entitled "Multimodal system supporting acoustic communication with computers" financed by the National Centre for Research and Development.

\section{References}

[1] P.J. Westervelt, Parametric Acoustic Array, J. Acoust. Soc. 35(4), 535-537 (1963).

[2] M. Yoneyama., J. Fujimoto, The audio spotlight: An application of nonlinear interaction of sound waves to a new type of loudspeaker design, J. Acoust. Soc. Am. 73(5), 1532-1536 (1983).

[3] M.F. Hamilton, D.T. Blackstock, Nonlinear Acoustics, Academic Press, San Diego 1998, Chap. 3.

[4] W. Gan, J. Yang, T. Kamakura, A review of parametric acoustic array in air, J. Applied Acoust 73(12), 1211-1219 (2012).
[5] T. Kamakura, K. Aoki, A Highly Directional Audio System Rusing Parametric Array in Air, 9th WESPAC, Seoul, 2006.

[6] T. Gudra, K. Opieliński, The Parametric Formation of Acoustic Waves in the Air by Using Ultrasonic Transducers, Revista de Acustica XXXII, Sevilla 2002.

[7] C. Shi, W. Gan, Development of a Parametric Loudspeaker: A Novel Directional Generation Technology, IEEE Potentials 29(6), 20-24 (2010).

[8] J. Croft, M. Spencer, J. Norris, Modulator processing for a parametric speaker system, Patent no. US6584205, 24.06.2003.

[9] T4010A Ultrasonic Transmitter, Nippon Ceramic, datasheet visited online (24.05.2014): http://www.nicera.co.jp/pro/ut/pdf/T4010A1(ENG).pdf

[10] F100B Ultrasonic Parametric Speaker Driving Circuit, Nippon Ceramic, datasheet visited online (24.05.2014): http://www.nicera.co.jp/pro/ut/ut-04e.html

[11] K. Miura, Ultrasonic Directive Speaker, Elektor Magazine, issue 3/2011, March 2011, pp. 56-60.

[12] FOCUS Fast Object-Oriented C++ Ultrasound Simulator. Michigan State University. Project homepage: http://www.egr.msu.edu/ fultras-web/index.php

[13] K. Żopatka, A. Czyżewski, Measurements of acoustic crosstalk cancellation efficiency in mobile listening conditions, IEEE Conf. on Signal Processing Algorithms, Architectures, Arrangements and Applications, Poznan, 2013, pp. 215- 219.
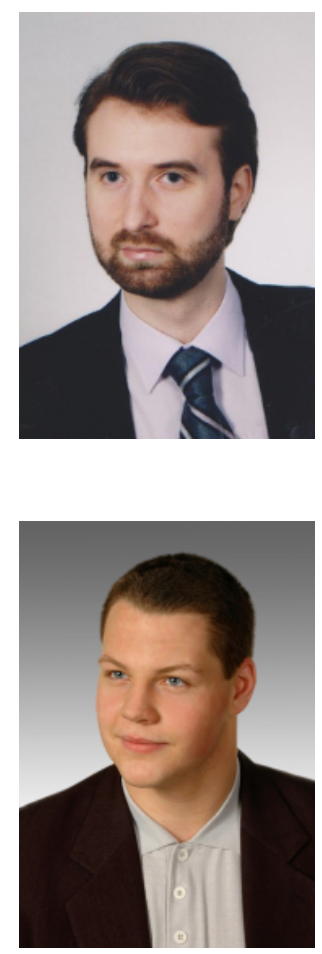

Piotr Bratoszewski was born in 1987 in Swiecie, Poland. In 2011 he graduated from the Multimedia Systems Department with an MS. degree. The subject of his thesis was related to feature extraction and classification algorithms in digital video. Currently he is a PhD student at the Multimedia Systems Department, and his research domain is mainly related to human-machine interfaces: automatic speech recognition and gesture recognition. Mr. Bratoszewski has co-authored in research papers related to automatic surveillance systems, automatic speech recognition and object tracking in video images.

Janusz Cichowski is a native of Gdansk, Poland. He received his MSc degree in Sound and Visual Engineering from Gdansk University of Technology in 2011. He graduated from the Multimedia Systems Department. The subject of his MSc thesis was related to digital watermarking and hiding image sensitive objects in surveillance systems with regard to privacy issues. At present he is a PhD student at the Multimedia Systems Department. He has co-authored research papers published and presented at conferences and related to privacy enhancement and anonymization for smart surveillance in the public domain. Mr. Cichowski is a member of the Polish Section of the Audio Engineering Society. 


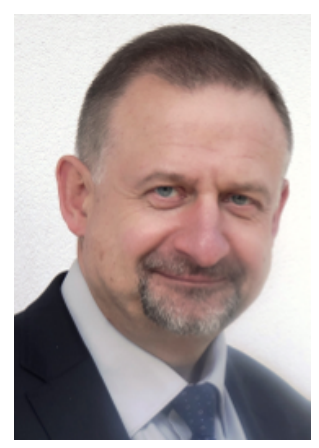

Andrzej Czyżewski is a native of Gdansk, Poland. He received his MSc degree in Sound Engineering from the Gdansk University of Technology in 1982, his PhD degree in 1987 and his DS. degree in 1992 from the AGH University of Science and Technology in Krakow. He joined the staff of the Sound Engineering Department of Gdansk University of Technology in 1984. In December 1999, the President of Poland granted him the title of Professor. In 2002, the Senate of his University approved him to the position of Full Professor. $\mathrm{He}$ is author of more than 400 research papers published in international journals or presented at congresses and conferences around the world. In 1991, he published a monograph devoted to digital audio operations, and in 1998 he published in Poland his book entitled "Digital Sound" which won him the Prize of Ministry of Education of Poland. He is also author of 10 Polish patents in the domain of computer science and 5 international patent applications. Prof. Czyżewski serves as Head of the Multimedia Systems Department of Gdansk University of Technology; Director of the Doctoral Studies (1994-2007) at the Faculty of Electronics, Telecommunications \& Informatics. He holds Fellowship of the Audio Engineering Society and he is a member of: IEEE, International Rough Set Society, and others. 